\title{
Simple Randomization
}

National Cancer Institute

\section{Source}

National Cancer Institute. Simple Randomization. NCI Thesaurus. Code C147144.

A type of randomization schema in which each subject has the same chance of being randomized into any one group as all other subjects. The assignment of the previous subject has no bearing on how the current subject will be assigned. 